\title{
Análisis de la recaudación del régimen impositivo simplificado ecuatoriano (RISE) y su importancia en los ingresos tradicionales del Presupuesto General del Estado. Periodo 2008-2017
}

\section{Analysis of the collection of the Ecuadorian simplified tax regime (RISE) and its importance in the traditional revenues of the General State Budget. 2008-2017 period}

Jorge Antonio Ruso León

Digna Priscila Villamar Ortiz

Adriana Rebeca Ordeñana Proaño

Edmundo Ricardo Contreras Chacón

Universidad Agraria del Ecuador, Ecuador

Autor para correspondencia: jruso@uagraria.edu.ec; pvillamar@uagraria.edu.ec; aordenana@uagraria.edu.ec; econtreras@uagraria.edu.ec

Fecha de recepción: 14 de abril de 2019 - Fecha de aceptación: 26 de agosto del 2019

Resumen: La informalidad laboral constituye una problemática extendida en el Ecuador y América Latina en general. Los gobiernos diseñan políticas con el fin de incentivar la formalización laboral, combatir la evasión tributaria e incrementar los ingresos presupuestarios. El Régimen Impositivo Simplificado ecuatoriano fue diseñado como un sistema sencillo y flexible que facilita el cumplimiento de las obligaciones fiscales de los pequeños empresarios fomentando la incorporación al mismo de los trabajadores informales. La investigación evalúa el éxito de este régimen tributario desde el punto de vista de su aporte a los ingresos del Presupuesto General del Estado y de la evolución de la participación de los ingresos reportados por este nuevo régimen en los Ingresos Tradicionales del presupuesto. Se comparó la recaudación presupuestada y la real con el nuevo régimen y su evolución, así como su participación dentro de los Ingresos Tradicionales del presupuesto. Finalmente, se verificó que la recaudación del nuevo sistema ha evolucionado de manera positiva incrementando su participación en los Ingresos Tradicionales del presupuesto a un ritmo mayor que el crecimiento total de este último indicador.

Palabras Claves: RISE; régimen tributario; presupuesto; informalidad; impuestos

\begin{abstract}
Labor informality is a widespread problem in Ecuador and Latin America in general. Governments design policies to encourage labor formalization, combat tax evasion and increase budget revenues. The Ecuadorian Simplified Tax Regime was designed as a simple and flexible system that facilitates compliance with the fiscal obligations of small entrepreneurs and encourages the incorporation of informal workers into the system. The investigation evaluates the success of this tax regime from the point of view of its contribution to the income of the General State Budget and the evolution of the participation of the income reported by this new regime in the Traditional Income of the budget. The budgeted and actual collections with the new regime were compared as well as its evolution and its participation within the Traditional Income of the budget. Finally, it was verified that the collection of the new system has evolved positively increasing its participation in the Traditional Income of the budget at a faster rate than the total growth of this last indicator.
\end{abstract}

Key Words: RISE; tax regime; budget; informality; taxes 


\section{Introducción}

La informalidad laboral y las medidas, estrategias o instrumentos, ya sean de política económica o tributaria, que se ponen en práctica con el fin de reducirla, conjuntamente con los perjuicios que se derivan de la misma, son temas de atención prioritaria por parte de los estados.

La importancia cada vez mayor que ha cobrado la economía informal tanto en los segmentos tradicionales de la economía como en los segmentos emergentes, sumada a la multiplicidad de formas en que la misma se manifiesta, según la Organización Internacional del Trabajo (2002), es razón suficiente para examinar este problema con el fin de diseñar políticas orientadas a la formalización laboral en las diferentes facetas en que esta tiene lugar.

El alto grado de las transferencias de bienes y servicios dentro de un país de manera informal, sobre todo en los países latinoamericanos es un serio problema social, la Organización Internacional del Trabajo (2018) refiere que la incidencia de la informalidad en la región sigue siendo generalizada y es una de las más elevadas del mundo. El Estado para solucionar estos inconvenientes establece diferentes acciones, algunas de ellas relacionadas con el campo tributario, a fin de que las mismas se constituyan en incentivos que despierten interés en el contribuyente de estos sectores para que de manera voluntaria formalicen su actividad, a la vez que se logra fomentar la cultura tributaria, según Diario El Universo (2008).

El Ecuador, según Aguirre \& Pazmiño (2011), tiene el problema de los comerciantes informales y personas que realizan actividades de manera independiente, sin comunicar a la Administración Tributaria la realización de su actividad y mucho menos pagar al Estado contribuciones por las rentas que perciben, aunque estos sectores informales, desde el punto de vista del volumen de sus ingresos, son menos representativos dentro de una nación en comparación con las grandes empresas, el monto anual de la recaudación de sus tributos no sería tan significativo, pero tampoco sería despreciable, por ello, el Gobierno Ecuatoriano patrocinó desde el año 2008, el llamado Régimen Impositivo Simplificado (RISE), pues no existía dentro de la legislación tributaria ecuatoriana, un sistema que regulara o explicara el tratamiento que debían tener los contribuyentes que realizaban transferencias de bienes y servicios y que poseían un negocio o local comercial; cayendo esta situación en una evasión tributaria, que con el régimen anteriormente citado, se erradicaría de una manera importante.

Esta evasión tributaria, que se pretende combatir con el sistema RISE, era significativa al momento de su creación en el año 2008, siendo, según Avilés (2007), un problema crítico en el ámbito tributario y económico, que genera efectos no deseados en el sistema económico y fiscal del país, dado el tamaño de la informalidad. Sin embargo, la formalización de negocios tiene trabas, expresa Revista Líderes (2015), y una de ellas lo es el costo que implica lo que desanima a los emprendedores, por ello, Avilés (2007) plantea que un sistema simplificado puede actuar como incentivo a la formalización de las empresas que operan al margen de la legalidad.

El 29 de diciembre del año 2007 mediante Registro Oficial se publicó la Ley de Equidad Tributaria del Ecuador, dentro de la cual nace el Régimen Impositivo Simplificado del Ecuador (RISE) siguiendo la tendencia experimentada por la mayoría de países latinoamericanos como: Argentina, Bolivia, Brasil, Colombia, México, Perú y Chile, donde se han implementado 
diversas fórmulas en la búsqueda de un eficiente y eficaz proceso de recaudación tributaria, no discriminador y que vaya en dirección opuesta al desarrollo de brechas sociales y económicas según expresan Ganga, Cabello \& Piñones (2014).

El motivo de realizar esta investigación es conocer si, en el período de estudio, se cumplió con los ingresos presupuestados por concepto del RISE, indagar sobre las causas de los incumplimientos, establecer la evolución de la participación del RISE dentro de los ingresos tradicionales y demostrar si el promedio de crecimiento de la recaudación del RISE excede el promedio de crecimiento de los Ingresos Tradicionales del PGE lo que permitiría determinar la importancia o el peso que ha ganado este tributo desde su aplicación.

Por tanto, el objetivo general de la presente investigación es:

- Establecer el cumplimiento y evolución de la recaudación del Régimen Impositivo Simplificado Ecuatoriano (RISE) y su importancia en los Ingresos Tradicionales del Presupuesto General del Estado (PGE) durante el período 2008-2017.

Asimismo, se determinan los siguientes objetivos específicos, los que se detallan a continuación:

- Compilar los valores recaudados por el RISE y los montos presupuestados por el mismo concepto en el PGE durante el periodo 2008-2017, asimismo, obtener los totales correspondiente a la ejecución de los Ingresos Tradicionales del PGE en el mismo período.

- Realizar la comparación entre la recaudación presupuestada y el monto real recaudado por concepto del RISE, con el fin de comprobar si se han logrado las metas planteadas y establecer las causas que hayan impedido cumplir dicha meta, si así fuera.

- Determinar la participación o proporción de la recaudación del RISE en el total de los Ingresos Tradicionales del PGE y revisar la tendencia de su evolución.

- Analizar la variación porcentual en la recaudación del RISE y los Ingresos Tradicionales del PGE evaluando la tendencia de esta variación en ambas variables y realizando una comparación de la dinámica de crecimiento de las mismas.

\section{Marco teórico}

La informalidad es un fenómeno complejo, a tenor de lo planteado por Serrano (2010), que también expresa que sus causas esconden problemáticas estructurales como la pobreza, la exclusión social, el desempleo y las desigualdades sociales. Este autor añade, que la informalidad debe ser estudiada como parte del complejo entramado de relaciones económicas y laborales que tiene lugar dentro de un sistema económico de múltiples dimensiones, una de las cuales es la tributaria.

Asimismo, Serrano (2010) expone que el término informalidad es difícil de definir, lo mismo se aplica a los trabajadores sin contrato formal que a aquellos que no están inmersos en los sistemas de seguridad social pública, también se aplica con frecuencia a los vendedores ambulantes, a los trabajadores a domicilio, etc. A efectos de la presente investigación se utiliza la 
definición de Molina, Rivadeneira, \& Rosero (2015), o sea, que trabajador informal es aquella persona con empleo que trabaja en empresas que no tienen Registro Único de Contribuyentes (RUC).

El régimen tributario, según Maldonado (2005), es la norma jurídica que regula las relaciones entre el Estado y demás entes acreedores de tributos, y quienes tienen obligaciones tributarias para con ellos. Por su parte, la Plataforma digital única del Estado Peruano (2019), define los regímenes tributarios como las categorías bajo las cuales una persona natural o jurídica que posee o va a iniciar un negocio debe estar registrada en los registros de la autoridad tributaria de un Estado en particular. El régimen tributario establece la manera en la que se pagan los impuestos y los niveles de pagos de los mismos. Puede optarse por uno u otro régimen dependiendo del tipo y el tamaño del negocio.

El RISE, se clasifica como un régimen tributario simplificado que para cumplir con la finalidad por la cual fue creado, pretende brindar facilidades a los pequeños contribuyentes, siguiendo la propuesta de Stiglitz (2000), de que un "buen" sistema tributario debe ser eficiente, sencillo y flexible, de tal manera que solo cancelen una cuota mensual y/o anual de acuerdo a su actividad económica, exonerándoles de la declaración del impuesto al valor agregado (IVA) y del impuesto a la renta (IR), además simplifica el llenado de los comprobantes de venta, de manera fácil y rápida, distinta a los otros contribuyentes, determinando que las personas que están en este sistema no deben de tener ingresos superiores a US\$ 60.000 dólares anuales.

Cabe indicar que dentro de este sistema existen ocho actividades distintas de comercio y servicios respectivamente, dependiendo de esto, el contribuyente pagará una cuota fija, el mismo que elegirá si la cancela de manera mensual o anual.

Por otra parte, el Presupuesto General del Estado (PGE) representa la estimación de los recursos financieros que tiene el país, Bermúdez (2015) lo define como una estructura que contiene los diferentes recursos financieros que tiene el Ecuador y que se aplican para un determinado periodo de tiempo; es decir, aquí están los Ingresos (venta de petróleo, recaudación de impuestos, etc.) pero también están los Gastos (de servicio, producción y funcionamiento estatal para educación, salud, vivienda, agricultura, seguridad, transporte, electricidad, etc. de acuerdo a las necesidades identificadas en los sectores y a la planificación de programas de desarrollo), según Ministerio de Economía y Finanzas (2019).

En la República del Ecuador el Presupuesto General del Estado presenta la siguiente clasificación para los ingresos, de acuerdo con el Ministerio de Economía y Finanzas (2019): permanentes y no permanentes, los permanentes son aquellos que se mantienen durante un período de tiempo, y son predecibles (IVA, ICE, IR, RISE, etc.), por su parte, los no permanentes son los ingresos no predecibles en el tiempo (ingresos petroleros, la venta de activos, los desembolsos de créditos, entre otros). Por su parte, el Observatorio Económico Social UNR (2015), clasifica los ingresos presupuestarios según su fuente en: tributarios y no tributarios, los primeros son los originados en la potestad que tiene el Estado de establecer gravámenes (IR, IVA, RISE y otros) mientras que los segundos son aquellos provenientes de fuentes no impositivas (tasas, regalías, derechos, alquileres, primas, multas, etc.). 
Dentro de los cuentas de ingresos permanentes del PGE está la subpartida denominada "Ingresos Tradicionales" donde se contabilizan los conceptos de ingresos tributarios de mayor peso, el IR y el IVA, entre otros impuestos de menor entidad, como el RISE.

\section{Metodología}

La hipótesis a probar en esta investigación es la siguiente:

"La recaudación tributaria del RISE cumplió o excedió los ingresos presupuestados en la cuenta o acápite correspondiente del PGE manteniendo a la vez una participación creciente y sostenida dentro de los Ingresos Tradicionales del mismo durante el período de estudio, evidenciándose el gradual incremento de su peso o importancia dentro de los mismos".

Para comprobar la anterior hipótesis se procedió a realizar una búsqueda y revisión de bases de datos en línea, para obtener la información de las recaudaciones tributarias del período analizado accediendo a la página WEB del SRI, a través del portal de internet del Ministerio de Economía y Finanzas se recopiló la información concerniente a la ejecución de los Ingresos Tradicionales del Presupuesto General del Estado.

Una vez compilada la información, esta se tabuló y se estableció una comparación para determinar la ejecución anual del presupuesto del RISE, asimismo se calculó el peso o porcentaje de participación de lo recaudado por concepto del RISE en el total de los Ingresos Tradicionales del Presupuesto General del Estado y se elaboraron gráficos para facilitar los análisis a realizar. (Ver Tabla 1 y Figura 1)

Tabla 1.

Montos presupuestados y recaudados del RISE, ejecución presupuestaria del RISE, ingresos tradicionales y participación del RISE dentro de los ingresos tradicionales, período 2008-2017. (En miles de dólares)

\begin{tabular}{|c|c|c|c|c|c|}
\hline Años & $\begin{array}{r}\text { Presupuesto } \\
\text { RISE }^{1} \\
\end{array}$ & $\begin{array}{r}\text { Recaudación } \\
\text { RISE }^{2}\end{array}$ & $\begin{array}{r}\text { \% ejecución } \\
\text { presupuestaria } \\
\text { del RISE } \\
\end{array}$ & $\begin{array}{r}\text { Total de } \\
\text { Ingresos } \\
\text { Tradicionales }^{3} \\
\end{array}$ & $\begin{array}{r}\text { \% participación del } \\
\text { RISE los Ing. } \\
\text { Tradicionales }\end{array}$ \\
\hline 2008 & $\$ 0$ & $\$ 396$ & & $\$ 6.508 .523$ & $0,0061 \%$ \\
\hline 2009 & $\$ 3.599$ & $\$ 3.666$ & $101,9 \%$ & $\$ 6.849 .788$ & $0,0535 \%$ \\
\hline 2010 & $\$ 5.819$ & $\$ 5.658$ & $97,2 \%$ & \$ 8.635.077 & $0,0655 \%$ \\
\hline 2011 & $\$ 6.200$ & $\$ 9.430$ & $152,1 \%$ & $\$ 9.815 .750$ & $0,0961 \%$ \\
\hline 2012 & $\$ 12.210$ & $\$ 12.170$ & $99,7 \%$ & \$12.129.980 & $0,1003 \%$ \\
\hline 2013 & $\$ 14.910$ & $\$ 15.120$ & $101,4 \%$ & \$ 15.499 .560 & $0,0976 \%$ \\
\hline 2014 & $\$ 19.670$ & $\$ 19.090$ & $97,1 \%$ & \$15.175.990 & $0,1258 \%$ \\
\hline 2015 & $\$ 20.000$ & $\$ 19.860$ & 99,3\% & \$16.030.600 & $0,1239 \%$ \\
\hline 2016 & $\$ 20.160$ & $\$ 18.870$ & $93,6 \%$ & $\$ 14.043 .230$ & $0,1344 \%$ \\
\hline 2017 & $\$ 22.580$ & $\$ 22.030$ & $97,6 \%$ & \$14.067.160 & $0,1566 \%$ \\
\hline
\end{tabular}




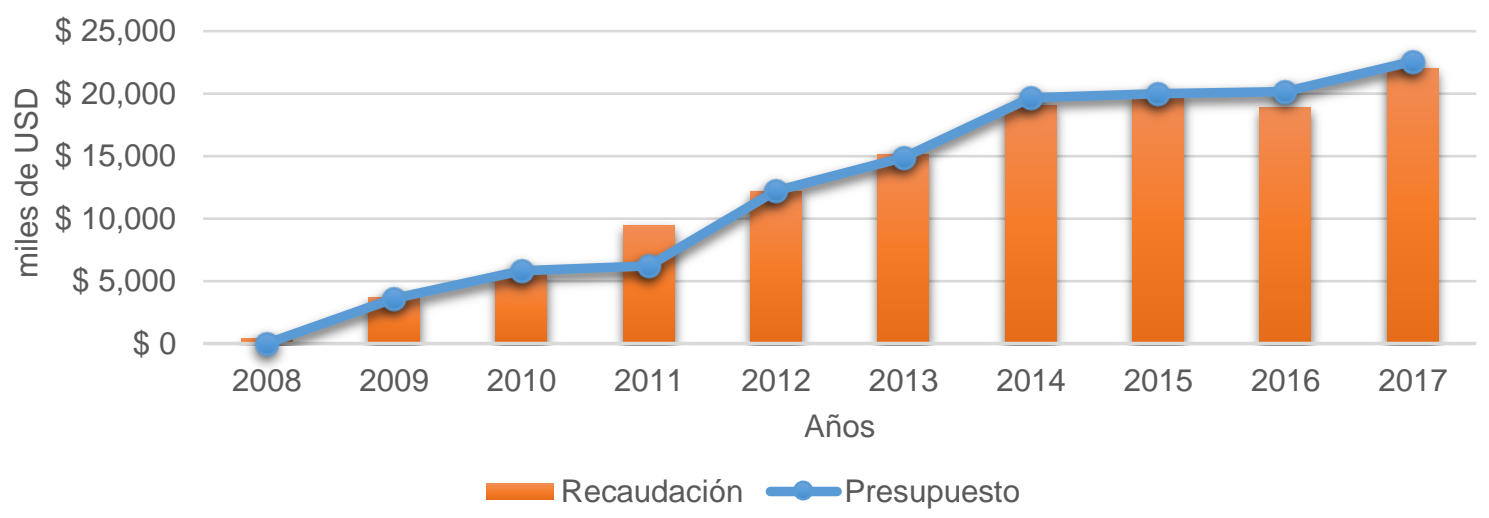

Figura 1 Presupuesto y ejecución de la recaudación del RISE en el periodo 2008-2017.

Fuente: Ministerio de Economía y Finanzas (2019).

Con el objetivo de comprobar si ha habido un desempeño creciente de la participación de la recaudación del RISE en el total de los Ingresos Tradicionales del PGE se aplicó una regresión lineal por $\mathrm{MCO}^{1}$ que permitió comprobar la tendencia de la serie de tiempo conformada por los porcentajes que representan la participación del RISE en el total de los Ingresos Tradicionales del Presupuesto General del Estado (Ver Figura 2). Previo a la aplicación de la regresión lineal se procedió a probar la normalidad de la serie de datos que sería ajustada linealmente, la estadística descriptiva de esta variable se aprecia en la Tabla 2, la cual se realizó con el fin de constatar si la misma se ajusta a una distribución normal.

Luego, para determinar la tendencia de las series de datos correspondientes a la variación porcentual de la recaudación del RISE y de los Ingresos Tradicionales del PGE, fue aplicada una regresión lineal por MCO a ambas variables hallándose la función lineal de mejor ajuste para ambas series con lo cual se pudo constatar la tendencia de esas series de datos (Ver Tabla 3 y Figura 3). La Tabla 2 muestra la estadística descriptiva de ambas variables donde se comprueba la normalidad de las mismas.

Tabla 2.

Estadística descriptiva.

\begin{tabular}{lrrr}
\hline & \multicolumn{3}{c}{ Variables } \\
\cline { 2 - 4 } Estadístico & $\begin{array}{r}\text { Participación del RISE } \\
\text { en el total de los Ingresos } \\
\text { Tradicionales del PGE }\end{array}$ & $\begin{array}{r}\text { Variación de la } \\
\text { recaudación del RISE }\end{array}$ & $\begin{array}{r}\text { Variación de los } \\
\text { Ingresos Tradicionales }\end{array}$ \\
\hline Frecuencia & 9 & $8^{(*)}$ & 9 \\
Media & 0,00105967 & 0,270439 & 0,0973912 \\
Varianza & $1,08461 \mathrm{E}-7$ & 0,0569827 & 0,0194677 \\
Desv. estándar & 0,000329335 & 0,23871 & 0,139527 \\
Mínimo & 0,000535 & $-0,049849$ & $-0,123974$ \\
Máximo & 0,001566 & 0,666667 & 0,277789 \\
Rango & 0,001031 & 0,716516 & 0,401763 \\
Asimetría & $-0,253786$ & 0,588426 & $-0,0439593$ \\
estandarizada & & & $-0,710752$ \\
Curtosis estandarizada & $-0,323198$ & $-0,168752$ & \\
\hline
\end{tabular}

${ }^{1}$ Mínimos cuadrados ordinarios 
Para las 3 variables analizadas se cumple la condición de normalidad en tanto que los coeficientes de asimetría y curtosis estandarizados están dentro del rango de -2 a +2 , que es el esperado en una variable que se distribuye normalmente lo

Condiciones de normalidad cual permite validar cualquier test estadístico basado en este supuesto. (*) En el

caso de la variable "Variación de la recaudación del RISE" se elimina la observación correspondiente al año 2009 por considerarse aberrante.

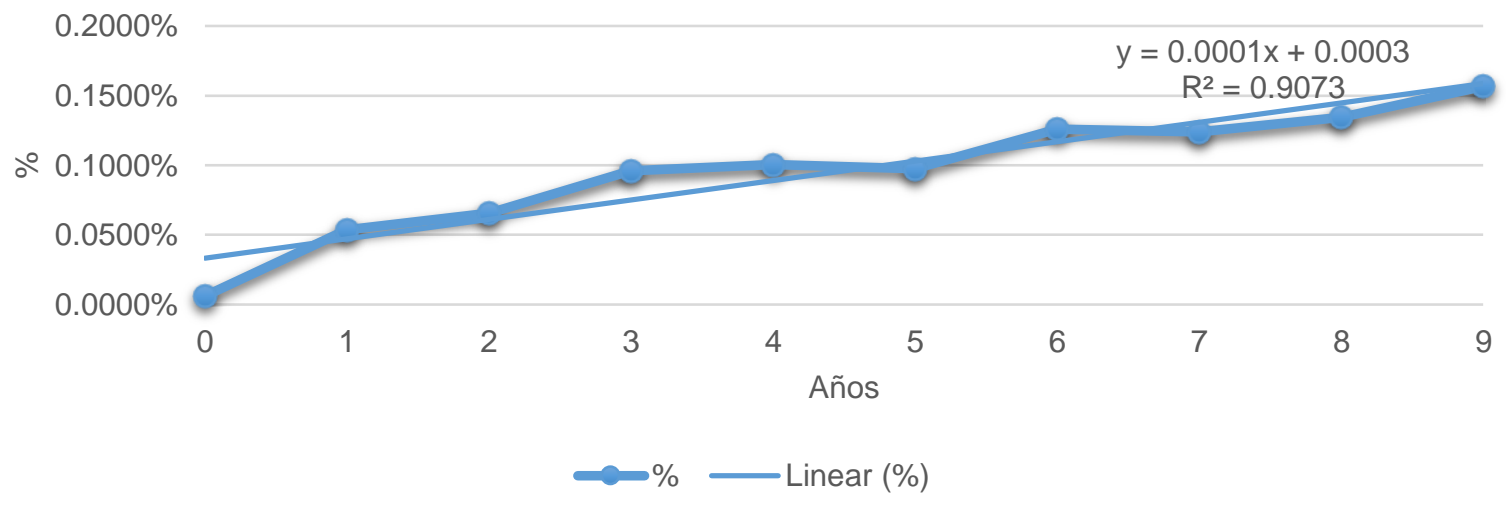

Figura 2 Porcentaje de las recaudaciones del RISE dentro del total de Ingresos Tradicionales del Presupuesto General del Estado (2008/año 0 - 2017/año 9) y recta de mejor ajuste.

Fuente: Ministerio de Economía y Finanzas (2019).

Seguidamente se ejecutó una prueba t de Student para las medias de las variables variación porcentual de la recaudación del RISE y variación porcentual de los Ingresos Tradicionales del PGE con el fin de probar la siguiente hipótesis:

\section{$\mathrm{H}_{0}: \boldsymbol{\mu}_{1} \leq \boldsymbol{\mu}_{2}$ \\ $H_{1}: \mu_{1}>\mu_{2}$}

Donde:

$\boldsymbol{\mu}_{1}$ : Media de la variación porcentual de la recaudación del RISE (8 observaciones, del 2010 al 2017 se descartó la observación correspondiente al 2008 por considerarse aberrante).

$\boldsymbol{\mu}_{2}$ : Media de la variación porcentual de los Ingresos Tradicionales del PGE (9 observaciones, del 2009 al 2017).

Tabla 3.

Variación de la recaudación del RISE y variación de los Ingresos Tradicionales del PGE, período 2008-2017.

\begin{tabular}{rrr}
\hline Años & $\begin{array}{r}\text { Variación de la } \\
\text { recaudación del } \\
\text { RISE }^{1}\end{array}$ & $\begin{array}{r}\text { Variación de los } \\
\text { Ingresos Tradic. } \\
\text { del PGE }^{2}\end{array}$ \\
\hline $\mathbf{2 0 0 8}$ & & \\
$\mathbf{2 0 0 9}$ & $825,8 \%$ & $5,2 \%$ \\
$\mathbf{2 0 1 0}$ & $54,3 \%$ & $26,1 \%$ \\
$\mathbf{2 0 1 1}$ & $66,7 \%$ & $13,7 \%$ \\
$\mathbf{2 0 1 2}$ & $29,1 \%$ & $23,6 \%$
\end{tabular}




\begin{tabular}{rrr}
\hline Años & $\begin{array}{r}\text { Variación de la } \\
\text { recaudación del } \\
\text { RISE }^{1}\end{array}$ & $\begin{array}{r}\text { Variación de los } \\
\text { Ingresos Tradic. } \\
\text { del PGE }\end{array}$ \\
\hline $\mathbf{2 0 1 3}$ & $24,2 \%$ & $27,8 \%$ \\
$\mathbf{2 0 1 4}$ & $26,3 \%$ & $-2,1 \%$ \\
$\mathbf{2 0 1 5}$ & $4,0 \%$ & $5,6 \%$ \\
$\mathbf{2 0 1 6}$ & $-5,0 \%$ & $-12,4 \%$ \\
$\mathbf{2 0 1 7}$ & $16,7 \%$ & $0,2 \%$ \\
\hline
\end{tabular}

Fuente: ${ }^{1}$ Servicio de Rentas Internas (2019) ${ }^{2}$ Ministerio de Economía y Finanzas (2019).

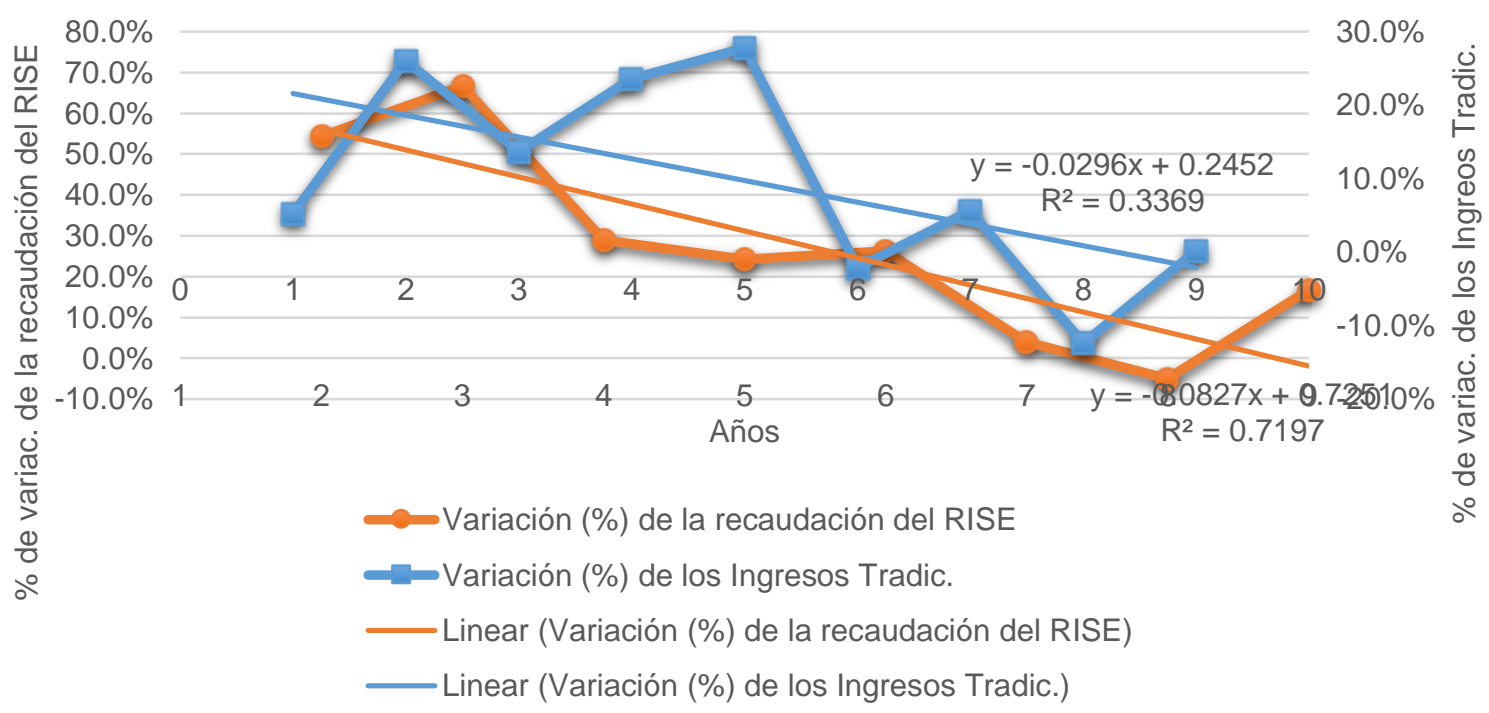

Figura 3 Variación porcentual de la recaudación del RISE (2010/año 2 - 2017/año 9) y variación porcentual de los Ingresos Tradicionales del PGE (2009/año 1- 2017/año 9) y las rectas de mejor ajuste de ambas series de datos. Fuente: Ministerio de Economía y Finanzas (2019).

Con esta prueba se pretende comprobar, de rechazarse $\mathrm{H}_{0}$, si durante el período de estudio la recaudación tributaria del RISE ha crecido a mayor ritmo que los Ingresos Tradicionales del PGE con lo cual se demostraría la creciente importancia que ha adquirido el RISE dentro de los ingresos presupuestarios.

\section{Resultados}

La Tabla 1 permite apreciar que para todo el período de estudio se obtiene una media de cumplimiento de la ejecución presupuestaria del RISE del 104,4\%, hay 4 años con sobrecumplimientos, mientras en los otros 6 años se aprecian distintos niveles de incumplimiento.

En los últimos años del estudio, del 2014 al 2017, se reportan incumplimientos, entre ellos el mayor de todo el período con el 93,6\%. En el 2012 y 2015 se reportan incumplimientos menores al $1 \%: 99,7 \%$ y $99,3 \%$ respectivamente.

Sin embargo, se observa en la Figura 1 la franca tendencia creciente que tiene la serie de datos correspondiente a la recaudación del RISE. 
Del análisis de la Tabla 1 y de la Figura 2 puede constatarse el peso o importancia que ha ido adquiriendo, la recaudación del RISE dentro de los Ingresos Tradicionales del PGE.

Por su parte, la Figura 2 visualiza la serie de tiempo correspondiente a la participación de la recaudación del RISE en los Ingresos Tradicionales del PGE la cual exhibe una tendencia creciente, confirmada por la pendiente positiva de la recta que mejor ajusta dicha serie.

Al analizar la Figura 3, donde se visualizan las series de datos correspondientes a las variaciones porcentuales de la recaudación del RISE y de los Ingresos Tradicionales del PGE, se comprueba la tendencia decreciente de ambas variables, afirmación corroborada por la pendiente negativa de las rectas que mejor ajustan las mismas.

La tendencia decreciente de la variación en la recaudación del RISE es evidencia del incremento del número de trabajadores informales que pasan a la formalidad quedando un número menor en la informalidad, lo cual se ve reflejado en la antes mencionada reducción en la variación del crecimiento de dicha recaudación.

Respecto a la variación del crecimiento de los Ingresos Tradicionales del PGE se observa en la Figura 3 que, a partir del 2014, las variaciones han sido las menores de todo el período de estudio, incluyendo dos años de decrecimiento (Ver Tabla 3), esto coincide con la situación económica de desaceleración de la economía ocurrida a partir del 2015 y provocada por la caída del precio internacional de los bienes básicos, entre ellos el petróleo, lo que afectó los resultados macroeconómicos ecuatorianos, situación que se mantiene hasta la fecha de cierre del presente estudio (Ver Figura 4) lo que se refleja en la evolución de los Ingresos Tradicionales del PGE.

Finalmente, con el fin de comprobar si la recaudación promedio del RISE durante el período de estudio ha sido estadísticamente superior a la media de crecimiento de los Ingresos Tradicionales del PGE, lo cual confirmaría el peso que ha ido adquiriendo el RISE en los ingresos presupuestarios, se procedió a realizar una prueba t de Student para medias para probar, como hipótesis nula, que la media de crecimiento de la recaudación del RISE ( $\left.\boldsymbol{\mu}_{1}\right)$ es menor o igual al promedio de crecimiento de los Ingresos Tradicionales del PGE $\left(\boldsymbol{\mu}_{2}\right)$.

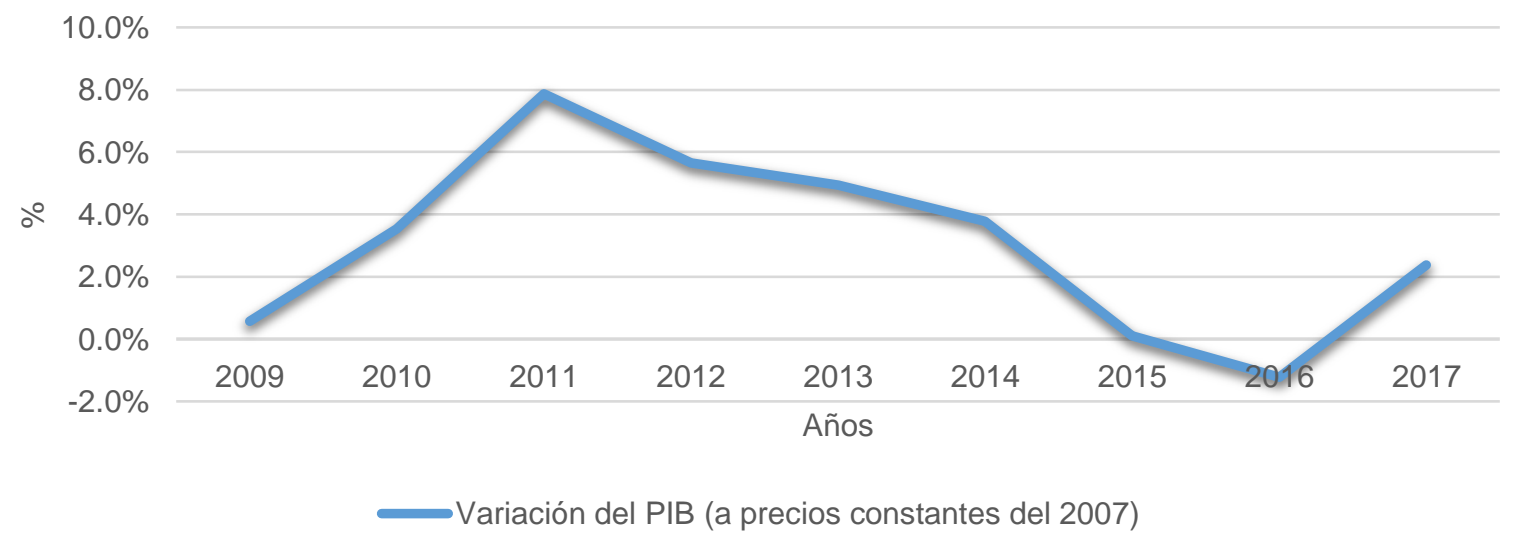

Figura 4 Variación del PIB ecuatoriano a precios constantes del 2007.

Fuente: Banco Central del Ecuador (2019). 
Los resultados de la prueba se evidencian en la Tabla 4, en ellos se aprecia que el p_valor para pruebas de una cola es de 0,0418998 , teniendo en cuenta que la prueba fue realizada con un nivel de significancia de 0.05 se procede a rechazar la hipótesis nula, $\mathbf{H}_{\mathbf{0}}: \boldsymbol{\mu}_{\mathbf{1}} \leq \boldsymbol{\mu}_{\mathbf{2}}$, por lo cual se concluye que hay evidencia estadística para afirmar que la media de crecimiento de la recaudación del RISE en el período de estudio fue mayor que el promedio de crecimiento de los Ingresos Tradicionales del PGE.

Tabla 4.

Resultado de la prueba t de Student para medias.

\begin{tabular}{|c|c|c|}
\hline & $\begin{array}{l}\text { Variación de } \\
\quad \text { la } \\
\text { recaudación } \\
\text { del RISE }\end{array}$ & $\begin{array}{c}\text { Variación de } \\
\text { los Ingresos } \\
\text { Tradicionales } \\
\text { del PGE }\end{array}$ \\
\hline Media & 0,2704392 & 0,097391307 \\
\hline Varianza & 0,05698259 & 0,019467662 \\
\hline Observaciones & 8 & 9 \\
\hline Varianza agrupada & 0,03697463 & \\
\hline Diferencia hipotética de las medias & 0 & \\
\hline Grados de libertad & 15 & \\
\hline Estadístico t & 1,85206489 & \\
\hline$P(T<=t)$ una cola & 0,0418998 & \\
\hline Valor crítico de $\mathbf{t}$ (una cola) & 1,75305036 & \\
\hline
\end{tabular}

Fuente: Elaboración por los autores.

\section{Conclusiones}

En el curso de la presente investigación se ha determinado que la ejecución presupuestaria de la recaudación del RISE a lo largo del período de análisis abarcado ha registrado variabilidad teniendo en cuenta que en 10 años se evidencian 4 sobrecumplimientos, 2 incumplimientos, los que no superan el $1 \%$ de lo presupuestado, y 4 incumplimientos, que superan el $1 \%$ del plan, correspondiendo el mayor incumplimiento al año 2016, sin embargo, éste no excede el $7 \%$ de lo planificado.

Cuando se revisa el porcentaje de cumplimiento promedio del período este alcanza el 104,4\% lo cual es positivo, sin embargo, durante los últimos 4 años del período todos estos corresponden a incumplimientos, lo cual pudiera achacarse a una deficiente planificación pues, si observamos la recaudación real, esta crece como media esos 4 años, pero no al ritmo que se previó en el presupuesto, el cual debió considerar en su concepción el período de desaceleración económica registrado desde el 2015 en el país y que puede constatarse observando la evolución del PIB a precios constantes del 2007 que se presenta en la Figura 4.

Otro elemento que confirma los supuestos anteriores apuntando directamente a los incumplimientos de los años 2016 y 2017 se refiere a que, luego del terremoto del 16 de abril del 2016, se aprobó la "Ley Orgánica de Solidaridad” en la que se dispone en su Disposición Transitoria Cuarta que a los contribuyentes del RISE domiciliados en las zonas afectadas por el terremoto se les exoneraba del 100\% del pago del impuesto desde Abril hasta Diciembre del 2016 y del 50\% del pago de impuesto correspondiente a todo el 2017 Registro Oficial (2016). 
A pesar de lo anterior, observando la Figura 1 se comprueba la evidente tendencia al crecimiento de las recaudaciones del RISE.

Respecto al peso o participación de las recaudaciones del RISE en el total de los Ingresos Tradicionales del PGE la Tabla 1 y la Figura 2 demuestran la tendencia creciente y sostenida de esta variable, corroborada por la pendiente positiva de la recta que mejor ajusta la serie de datos correspondiente a la variable. Esta tendencia apenas fue afectada por el período de ralentización económica que se verifica a partir del 2015.

En referencia a la situación de las series de tiempo correspondientes a la variación de la recaudación del RISE y de los Ingresos Tradicionales del PGE, se observó en la Figura 3 una tendencia decreciente en ambas variables, la cual se infiere, en el caso de la recaudación del RISE, a partir de que en cada período se integran al sistema un mayor número de contribuyentes quedando menos personas en la informalidad por incluirse lo que reduce el potencial de crecimiento.

En cuanto a la variación de los Ingresos Tradicionales del PGE, la caída de su crecimiento se produce, principalmente, por la desaceleración económica que registra el país a partir del 2015, según se aprecia en la Figura 4. De lo anterior puede concluirse que a lo largo del período de estudio para ambas series de datos se observa una caída en sus razones de crecimiento que se agudizan hacia el final del mismo y que se explican por los hechos antes expuestos.

Finalmente, la comparación de las medias de la variación de la recaudación del RISE y la variación de los Ingresos Tradicionales del PGE mediante la prueba t de Student realizada y cuyos resultados se muestran en la Tabla 4, permite concluir que el promedio de crecimiento de la recaudación del RISE es mayor, con significancia estadística, a la media de crecimiento de los Ingresos Tradicionales del PGE lo cual demuestra el creciente peso o importancia que ha adquirido el RISE dentro de los ingresos presupuestarios durante el período analizado.

Se concluye que la hipótesis de la investigación se confirma, pues en el período estudiado se cumple, de manera general, la recaudación presupuestada del RISE, se constata su participación creciente y sostenida en los Ingresos Tradicionales del PGE y se comprueba la gradual importancia que va adquiriendo la recaudación del RISE dentro de los ingresos del presupuesto.

\section{Bibliografía}

Aguirre, A., \& Pazmiño, I. (09 de 2011). Estudio comparativo de la evolución del RISE en el Ecuador y su aporte al Presupuesto General del Estado durante los años 2008 al 2010. Obtenido de Repositorio Institucional Universidad Politécnica Salesiana: https://dspace.ups.edu.ec/bitstream/123456789/1773/14/UPS-GT000228.pdf

Avilés, J. M. (2007). Implantación de un modelo de sistema simplificado para ecuador. Características del Modelo y Plan de Implantación. Fiscalidad Ed. No. 1, 61-99.

Banco Central del Ecuador. (2019). Información Estadística Mensual. Obtenido de Banco Central del Ecuador: https://contenido.bce.fin.ec/home1/estadisticas/bolmensual/IEMensual.jsp

Bermúdez, R. S. (08 de 2015). El Impuesto al Valor Agregado y su incidencia financiera en el Presupuesto General del Estado durante los periodos fiscales 2007 - 2012. Obtenido de 
Repositorio Universidad de Guayaquil: http://repositorio.ug.edu.ec/bitstream/redug/8488/1/Tesis\%20Final\%20Maestria.pdf

Diario El Universo. (28 de 07 de 2008). Los negocios serán inscritos para RISE. Obtenido de Diario El

Universo: https://www.eluniverso.com/2008/07/28/0001/9/6E6B845780AF4F17B3B836D8051296 92.html

Ganga, F., Cabello, X., \& Piñones, M. A. (06 de 2014). Estado e impuestos indirectos en Chile y Latinoamerica: análisis del período 2000 - 2011. Obtenido de Revista "Visión de Futuro" Vol. 18 No. 1: http://www.scielo.org.ar/scielo.php?script=sci_arttext\&pid=S166887082014000100003

Maldonado, H. (2005). El Régimen Tributario - Generalidades. Obtenido de Derecho Ecuador.com: https://www.derechoecuador.com/el-reacutegimen-tributario-generalidades

Ministerio de Economía y Finanzas. (2019). Ejecución Presupuestaria. Obtenido de Ministerio de Economía y Finanzas: https://www.finanzas.gob.ec/ejecucion-presupuestaria/

Ministerio de Economía y Finanzas. (2019). El Presupuesto General del Estado. Obtenido de Ministerio de Economía y Finanzas: https://www.finanzas.gob.ec/el-presupuesto-generaldel-estado/

Ministerio de Economía y Finanzas. (2019). Ingresos - Principios. Obtenido de Ministerio de Economía y Finanzas: https://www.finanzas.gob.ec/ingresos-principios/

Molina, A., Rivadeneira, A., \& Rosero, J. (2015). Actualización metodológica: el empleo en el sector informal. Revista de Estadística y Metodologías, 55-62. Obtenido de Instituto Nacional de Estadísticas.

Observatorio Económico Social UNR. (16 de 08 de 2015). Ingresos Públicos. Obtenido de Observatorio Económico Social Universidad Nacional de Rosario: http://www.observatorio.unr.edu.ar/ingresos-publicos/

Organización Internacional del Trabajo. (2002). El trabajo decente y la economía informal. Ginebra: OIT - Naciones Unidas.

Organización Internacional del Trabajo. (2018). Perspectivas sociales y del empleo en el mundo. Tendencias 2018. Ginebra: OIT - Naciones Unidas.

Plataforma digital única del Estado Peruano. (2019). Regímenes tributarios. Obtenido de Comercio, Negocio y Emprendimiento: https://www.gob.pe/280-regimenes-tributarios

Registro Oficial. (2016). Ley Orgánica de Solidaridad y de Corresponsabilidad Ciudadana para la Reconstrucción. Obtenido de Registro Oficial: https://www.registroficial.gob.ec/

Revista Líderes. (26 de 07 de 2015). La informalidad laboral, una condición arraigada en el Ecuador. Obtenido de Revista Líderes: https://www.revistalideres.ec/lideres/informalidadlaboral-condicion-ecuador.html

Serrano, A. (2010). Análisis de la Informalidad en Ecuador: Recetas Tributarias para su Gestión. Fiscalidad Ed. No. 4, 129-261.

Servicio de Rentas Internas. (2019). Estadísticas generales de recaudación. Obtenido de Servicio de Rentas Internas: http://www.sri.gob.ec/web/guest/estadisticas-generales-derecaudacion

Stiglitz, J. E. (2000). La Economía del Sector Público 3ra. Ed. Barcelona: Antoni Bosch, editor, S.A. 